\title{
Meta-analysis of oxaliplatin-based versus fluorouracil-based neoadjuvant chemoradiotherapy and adjuvant chemotherapy for locally advanced rectal cancer
}

\author{
Xing-Li Fu ${ }^{3, *}$, Zheng Fang ${ }^{2, *}$, Liang-Hui Shu ${ }^{4, *}$, Guo-Qing Tao ${ }^{1}$, Jian-Qiang Wang ${ }^{5}$, \\ Zhi-Lian Rui ${ }^{6}$, Yong-Jie Zhang ${ }^{2}$, Zhi-Qiang Tian ${ }^{1}$ \\ ${ }^{1}$ Department of General Surgery, Wuxi People's Hospital Affiliated Nanjing Medical University, Wuxi 214023, China \\ ${ }^{2}$ Department of Biliary Surgery, The Eastern Hepatobiliary Surgery Hospital, Second Military Medical University, Shanghai \\ 200438, China \\ ${ }^{3}$ Health Science Center, Jiangsu University, Zhenjiang, Jiangsu 212001, China \\ ${ }^{4}$ Department of Nephrology and Endocrinology, The 101st Hospital of Chinese PLA (Wuxi Taihu Hospital), Wuxi 214044, China \\ ${ }^{5}$ The Second People's Hospital of Jintan District, Changzhou, Jiangsu 213200, China \\ ${ }^{6}$ The People's Hospital of Liyang, Changzhou, Jiangsu 213300, China \\ *These authors contributed equally to this work
}

Correspondence to: Zhi-qiang Tian, email: zhiqiangtiann@163.com Yong-jie Zhang, email: 13951574216@163.com

Keywords: rectal cancer, neoadjuvant chemoradiotherapy, adjuvant chemotherapy, oxaliplatin, meta-analyses

Received: November 22, 2016

Accepted: February 22, 2017

Published: March 11, 2017

Copyright: Fu et al. This is an open-access article distributed under the terms of the Creative Commons Attribution License (CC-BY), which permits unrestricted use, distribution, and reproduction in any medium, provided the original author and source are credited.

\section{ABSTRACT}

A meta-analysis was conducted to compare oxaliplatin-based with fluorouracilbased neoadjuvant chemoradiotherapy and adjuvant chemotherapy for locally advanced rectal cancer. MEDLINE, EMBASE and CENTRAL were systematically searched for relevant randomized controlled trials (RCTs) until January 31 2017. Review Manager (version 5.3) was used to analyze the data. Dichotomous data were calculated by odds ratio (OR) with 95\% confidence intervals (CI). A total of 8 RCTs with 6103 stage II or III rectal cancer patients were analyzed, including 2887 patients with oxaliplatin+fluorouracil regimen and 3216 patients with fluorouracil alone regimen. Compared with fluorouracil-based regimen group, oxaliplatin-based regimen group attained higher pathologic complete response $(O R=1.29,95 \% \mathrm{CI}: 1.12-1.49$, $P=0.0005)$ and 3-year disease-free survival (OR $=1.15,95 \%$ CI: $0.93-1.42$, $P=0.21)$, but suffered greater toxicity (OR $=2.07,95 \%$ CI: $1.52-2.83, P<0.00001)$. Also, there were no significant differences between two regimens in sphincter-sparing surgery rates $(O R=0.94,95 \% C I: 0.83-1.06, P=0.33)$, 5 -year disease-free survival (OR $=1.15,95 \% \mathrm{CI}: 0.93-1.42, P=0.21$ ) and overall survival (3-year, $O R=1.14$, 95\% CI: 0.98-1.34, $P=0.09$; 5-year, OR = 1.06, 95\% CI: 0.78-1.44, $P=0.70$ ). In conclusion, the benefits of adding oxaliplatin to fluorouracil-based neoadjuvant chemoradiotherapy and adjuvant chemotherapy for locally advanced rectal cancer remains controversial, and cannot be considered a standard approach.

\section{INTRODUCTION}

Rectal cancer is a common and lethal disease. In Europe, 342,137 cases colorectal cancer are diagnosed in 2012 [1], and rectal cancer represents about $27 \%$ to $58 \%$ of cases [2]. In the United States, approximate 39,910 new cases of rectal cancer are diagnosed in 2017 [3]. Surgical resection is the cornerstone of curative therapy for rectal cancer [4]. However, about $55 \%$ of patients with rectal cancer are diagnosed at stage II or III [5], and only patients with early stage rectal cancer can attain a high cure rate by surgery [6]. It is a multidisciplinary approach of treatment for rectal cancer, preoperative and postoperative staging are of crucial importance for patients with stage II or III rectal cancer [7]. Several randomized clinical trials demonstrate that preoperative chemoradiation or short-course radiotherapy improves outcomes in locally advanced rectal cancer [8-11]. Preoperative chemoradiotherapy (or short- 
course radiotherapy alone) followed by total mesorectal excision surgery have markedly reduced local recurrence rates in stage II or III rectal cancer to well below $10 \%$ at 5 years in recent trials $[8,9,12]$. Therefore, this approach is considered one of the standard treatment strategy, especially for locally advanced rectal cancer. Moreover, postoperative chemotherapy is controversial for rectal cancer patients with preoperative chemoradiotherapy and surgery. The benefit of postoperative chemotherapy in rectal cancer patients undergoing preoperative chemoradiotherapy is uncertainty [13-16], although most oncologists recommend it and the majority of patients receive it in United States [17]. Actually, the use of preoperative chemoradiotherapy and postoperative chemotherapy varies among different treatment centers $[18,19]$. It is time to look for moreeffective systemic treatments.

Fluorouracil-based regimen is considered as a standard approach during neoadjuvant chemoradiotherapy and adjuvant chemotherapy in stage II or III rectal cancer $[11,20,21]$. Fluorouracil-based regimen mainly includes infusions of fluorouracil/leucovorin and oral daily capecitabine [11]. But, fluorouracil-based chemoradiotherapy has no impact on distant metastasis that remain in the $30 \%$ range [22]. Oxaliplatin has been adopted as a standard regimen of adjuvant chemotherapy of stage III colon cancer, and adding oxaliplatin to fluorouracil/leucovorin can improve therapeutic efficacy [23-26]. Nevertheless, the benefit of oxaliplatin-based neoadjuvant chemoradiotherapy and adjuvant chemotherapy in stage II or III rectal cancer remains unclear. There are at least eight randomized trials [27-36] investigate the effect of oxaliplatin-based neoadjuvant chemoradiotherapy and adjuvant chemotherapy for stage II or III rectal cancer. However, the efficacy data are controversial. All trials demonstrated that toxicity is clearly worse compared with chemoradiotherapy with a fluoropyrimidine alone and that efficacy is not yet proven. The question remains: if the addition of oxaliplatin in standard neoadjuvant chemoradiotherapy and adjuvant chemotherapy treatment regimen in stage II or III rectal cancer can provide a better survival although with higher toxicity.

There is no consensus on whether the addition of oxaliplatin in neoadjuvant chemoradiotherapy and adjuvant chemotherapy is benefit for patients with stage II or III rectal cancer. The article by Rödel et al. [33] in Lancet Oncology described the results of the latest German study in 2015. A systematic overview by Bujko et al. [19] in 2015 assessed this issue. However, not all eight trials more details were included in that meta-analysis. Hence, we conducted a more updated and better systematic review and meta-analysis on this controversial issue.

\section{RESULTS}

\section{Study selection}

A total of 853 potential abstracts were identified after deleting out duplication in extensive literature search of electronic database and manual approach until January 31 2017. 804 articles were further ruled out after scanning the title/abstract according to the inclusion and exclusion criteria of this meta-analysis. As for full-text of the remaining 49 articles were subjected to identify. Furthermore, 41 additional articles were ruled out for the reasons described in Figure 1. Finally, 8 RCTs published between 2011 and 2016 were included in quantitative synthesis in this meta-analysis. Figure 1 demonstrates a flow diagram of the detailed selection process.

\section{Study quality assessment}

Risk of bias assessment was adhered to Cochrane Collaboration's tool, because the included 8 studies were all randomized comparative studies. Figure 2 showed the risk of bias for each study (Figure 2). In all of 8 studies, sequence generation and allocation concealment were randomized, incomplete outcome data and selective reporting were low risk of bias, but healthcare provider and participants were not blinded (Figure 3). Risk of bias of outcome assessment was unclear, due to the outcome data gatherers were not blinded (Figure 3). Accordingly, risk of bias of the included studies in this meta-analysis was low.

\section{Study characteristics}

This meta-analysis included 8 RCTs [27-36] that were conducted in Germany (2), US (1), France (1), Korea (1), Italy (1), and China (2). The sample size of all studies were greater than 200 participants. Overview of the 8 included studies of this meta-analysis was shown in Table 1. This meta-analysis enrolled 6103 patients with stage II/ III rectal cancer, including 2887 patients with oxaliplatinbased regimen and 3216 patients with fluorouracil-based regimen. All participants were consecutively enrolled in the statement of studies. Baseline characteristics of these studies were summarized in Table 2.

\section{Synthesis of results}

\section{Disease-free survival}

Disease-free survival was the primary endpoint in most studies. There were 7 studies [27-33, 35, 36] comparing 3-year disease-free survival rate between oxaliplatin-based regimen and fluorouracil-based regimen of neoadjuvant chemoradiotherapy and adjuvant chemotherapy for rectal cancer. Heterogeneity was low among the studies $\left(P=0.25, I^{2}=23 \%\right)$, so the fixed effect model was used to pool the outcomes. The result $(\mathrm{OR}=1.13 ; 95 \% \mathrm{CI}=1.01$ to $1.27 ; P=0.04$ ) indicated that 3 -year disease-free survival was significant difference between two groups (Figure 4).

In addition, there were 2 trials [27, 31] comparing 5 -year disease-free survival rate between two groups. Heterogeneity was none among the studies $(P=0.99$, $I^{2}=0 \%$ ), so the fixed effect model was used to pool the 
outcomes. The result $(\mathrm{OR}=1.15 ; 95 \% \mathrm{CI}=0.93$ to 1.42 ; $P=0.21)$ inferred that 5 -year disease-free survival was no significant difference between two groups (Figure 5).

\section{Overall survival}

There were 6 studies $[27,28,31,33,35,36]$ compared 3-year overall survival rate between oxaliplatinbased regimen group and fluorouracil-based regimen group. Heterogeneity was none among the studies $(P=0.99$, $I^{2}=0 \%$ ), so the fixed effect model was used to pool the outcomes. The result $(\mathrm{OR}=1.14 ; 95 \% \mathrm{CI}=0.98$ to 1.34 ; $P=0.09$ ) suggested that 3 -year overall survival was no significant difference between two groups (Figure 6).

Moreover, 2 studies [27, 31] compared the 5-year overall survival rate between two groups. Heterogeneity was moderate among the studies $\left(P=0.13, I^{2}=57 \%\right)$, so the random effect model was used to pool the outcomes.
The result ( $\mathrm{OR}=1.06 ; 95 \% \mathrm{CI}=0.78$ to $1.44 ; P=0.70)$ implied that 5 -year overall survival was no significant difference between two groups (Figure 7).

\section{Pathologic complete response}

Pathologic complete response is an indication of efficacy with regard to oncological outcomes. 6 studies [27-34] compared the pathologic complete response between oxaliplatin-based regimen group and fluorouracilbased regimen group. Heterogeneity was moderate among the studies $\left(P=0.13, I^{2}=41 \%\right)$, so the fixed effect model was used to pool the outcomes. The result $(\mathrm{OR}=1.29 ; 95 \% \mathrm{CI}=1.12$ to $1.49 ; P=0.0005)$ indicated that oxaliplatin-based regimen group attained higher pathologic complete response than fluorouracil-based regimen group (Figure 8).

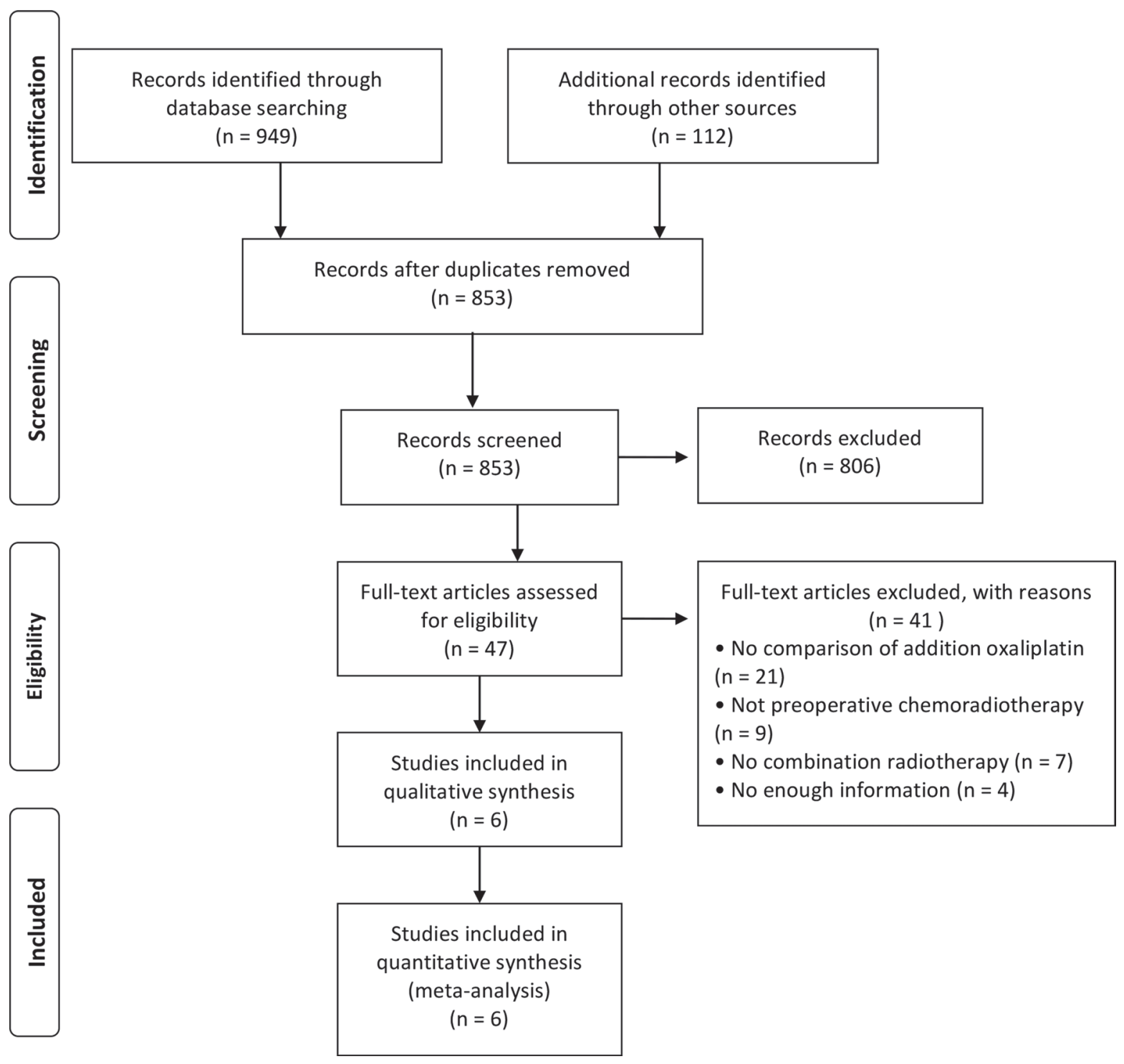

Figure 1: Flow diagram showing the selection of studies for inclusion in this meta-analysis. 


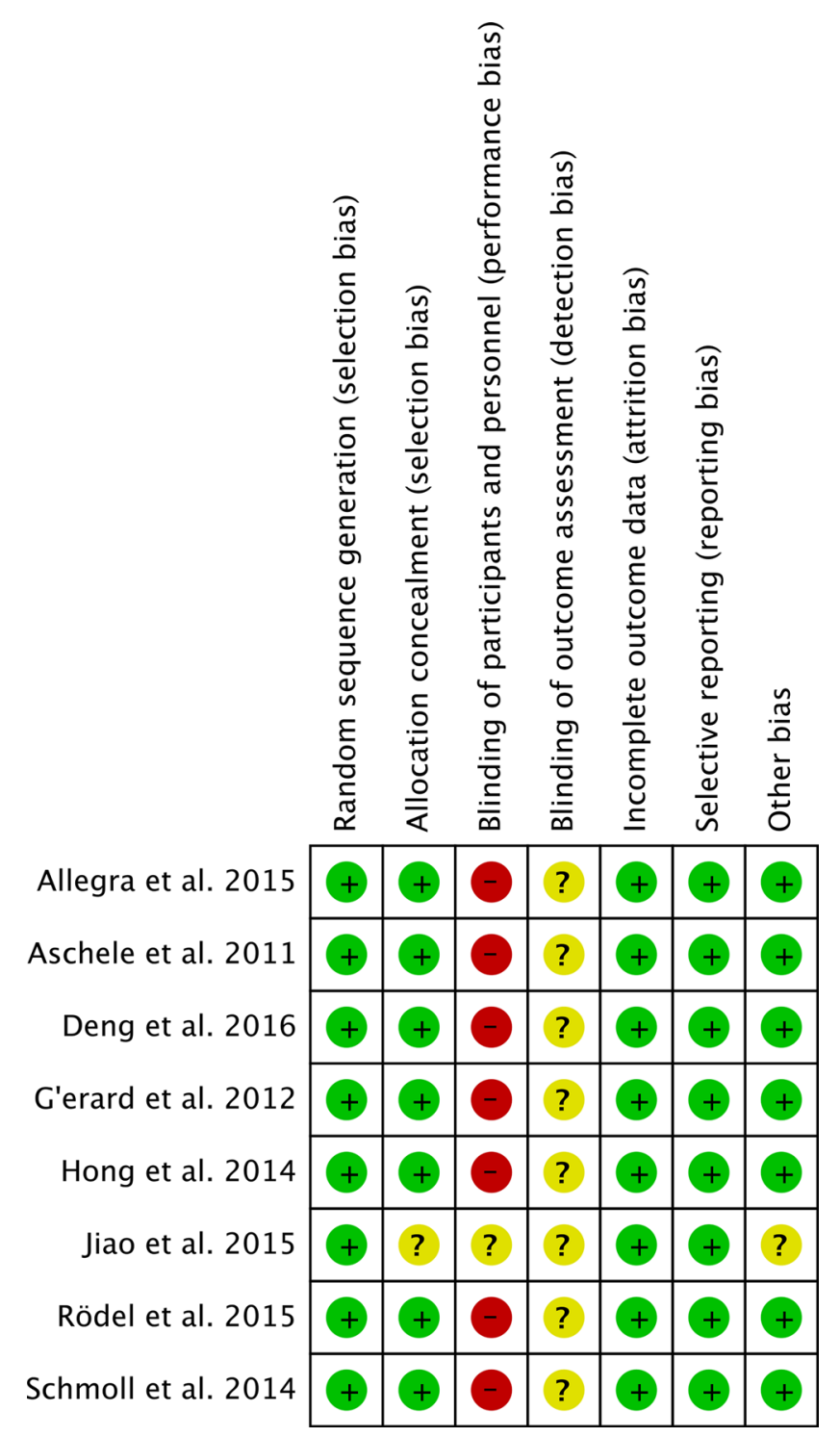

Figure 2: Summary of risk of bias for each selected study assessed by cochrane collaboration's tool.

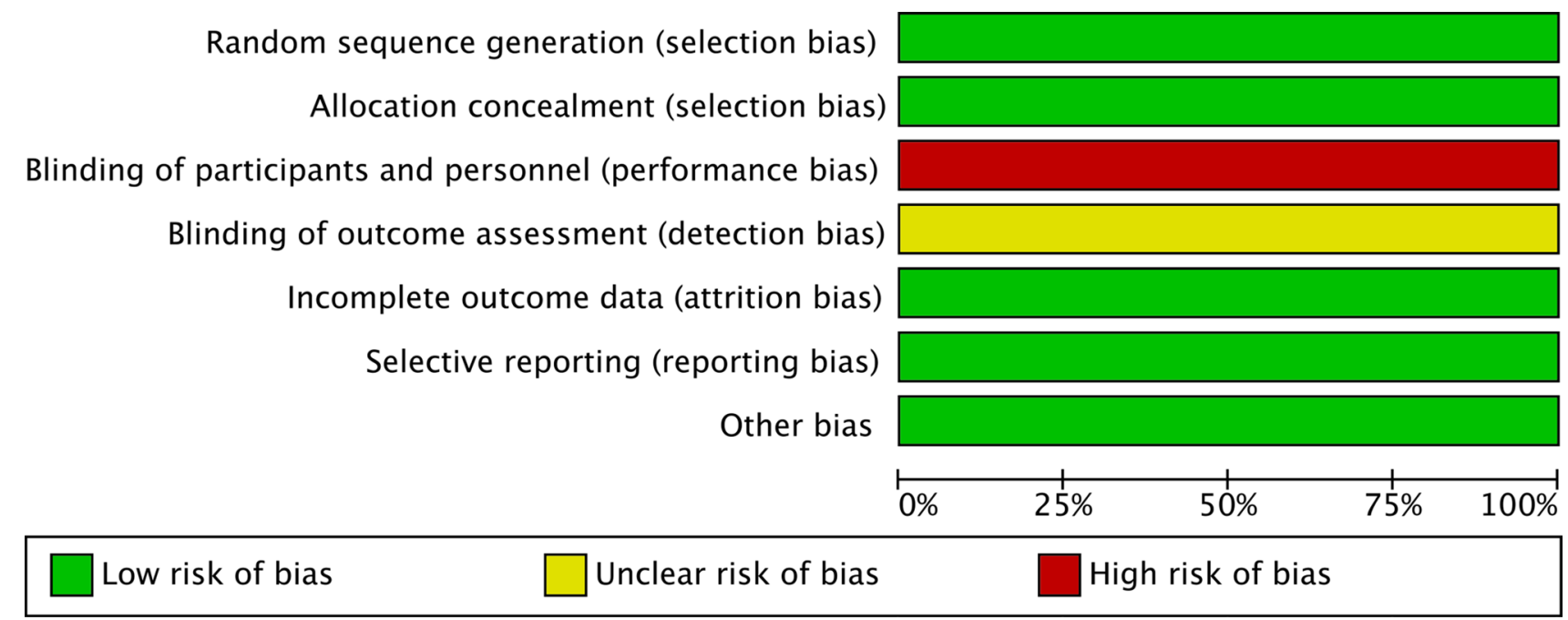

Figure 3: Risk of bias graph about each risk of bias item presented as percentages across all selected studies. 
Table 1: Overview of the included randomized control trials of this meta-analysis

\begin{tabular}{|c|c|c|c|c|c|c|}
\hline \multirow{2}{*}{$\begin{array}{l}\text { Studies (Author, year, } \\
\text { country) }\end{array}$} & \multirow{2}{*}{ Trials } & \multirow{2}{*}{ Design } & \multicolumn{2}{|c|}{ Chemotherapy regimens } & \multirow{2}{*}{ Radiation } & \multirow{2}{*}{$\begin{array}{c}\text { Follow Up } \\
\text { (median) }\end{array}$} \\
\hline & & & Treatment Group & Control Group & & \\
\hline $\begin{array}{l}\text { Allegra et al. [31, 32], } \\
2015 \text {, US }\end{array}$ & NSABP R-04 & $\begin{array}{l}\text { Multicenter, Open-Label, Randomized, Phase } \\
\text { III }\end{array}$ & $\begin{array}{l}\text { Preoperative: } \mathrm{OX}+\mathrm{FU} / \mathrm{CAPE} \\
\text { Postoperative: Not specified }\end{array}$ & $\begin{array}{l}\text { Preoperative: FU/CAPE } \\
\text { Postoperative: Not specified }\end{array}$ & $45 \mathrm{~Gy}$ & / \\
\hline $\begin{array}{l}\text { Aschele et al. [27]., } \\
\text { 2011, Italy }\end{array}$ & STAR-01 & $\begin{array}{l}\text { Multicenter, Open-Label, Randomized, Phase } \\
\text { III }\end{array}$ & $\begin{array}{l}\text { Preoperative: } \mathrm{OX}+\mathrm{FU} \\
\text { Postoperative: } \mathrm{FU}\end{array}$ & $\begin{array}{l}\text { Preoperative: FU } \\
\text { Postoperative: FU }\end{array}$ & $50.4 \mathrm{~Gy}$ & $105.6 \mathrm{~m}$ \\
\hline $\begin{array}{l}\text { Deng et al. }[34] \text {, } \\
2016 \text {, China }\end{array}$ & FOWARC & $\begin{array}{l}\text { Multicenter, Open-Label, Randomized, Phase } \\
\text { III }\end{array}$ & $\begin{array}{l}\text { Preoperative: } \mathrm{OX}+\mathrm{FU} \\
\text { Postoperative: } \mathrm{OX}+\mathrm{FU}\end{array}$ & $\begin{array}{l}\text { Preoperative: FU } \\
\text { Postoperative: FU }\end{array}$ & $46-50.4 \mathrm{~Gy}$ & / \\
\hline $\begin{array}{l}\text { Hong et al. [35], } \\
\text { 2014, Korea }\end{array}$ & ADORE & Multicenter, Open-Label, Randomized, Phase II & $\begin{array}{l}\text { Preoperative: } \mathrm{OX}+\mathrm{FU} \\
\text { Postoperative: } \mathrm{FU}\end{array}$ & $\begin{array}{l}\text { Preoperative: FU } \\
\text { Postoperative: FU }\end{array}$ & NA & $38.2 \mathrm{~m}$ \\
\hline $\begin{array}{l}\text { G'erard et al. }[28,29] \text {, } \\
\text { 2012, France }\end{array}$ & ACCORD 12/0405 & $\begin{array}{l}\text { Multicenter, Open-Label, Randomized, Phase } \\
\text { III }\end{array}$ & $\begin{array}{l}\text { Preoperative: } \mathrm{OX}+\mathrm{CAPE} \\
\text { Postoperative: } \mathrm{FU}\end{array}$ & $\begin{array}{l}\text { Preoperative: CAPE } \\
\text { Postoperative: FU }\end{array}$ & 45-50Gy & $36.8 \mathrm{~m}$ \\
\hline $\begin{array}{l}\text { Jiao et al. }[36] \text {, } \\
\text { 2015, China }\end{array}$ & l & $\begin{array}{l}\text { Single-center, Open-Label, } \\
\text { Randomized }\end{array}$ & $\begin{array}{l}\text { Preoperative: } \mathrm{OX}+\mathrm{CAPE} \\
\text { Postoperative: } \mathrm{OX}+\mathrm{FU}\end{array}$ & $\begin{array}{l}\text { Preoperative: } \mathrm{CAPE} \\
\text { Postoperative: } \mathrm{OX}+\mathrm{FU}\end{array}$ & $50.0 \mathrm{~Gy}$ & $48.7 \mathrm{~m}$ \\
\hline $\begin{array}{l}\text { Rödel et al. [33], } \\
\text { 2015, Germany }\end{array}$ & $\begin{array}{l}\mathrm{CAO} / \mathrm{ARO} / \\
\mathrm{AIO}-04\end{array}$ & $\begin{array}{l}\text { Multicenter, Open-Label, Randomized, Phase } \\
\text { III }\end{array}$ & $\begin{array}{l}\text { Preoperative: } \mathrm{OX}+\mathrm{FU} \\
\text { Postoperative: } \mathrm{OX}+\mathrm{FU}\end{array}$ & $\begin{array}{l}\text { Preoperative: FU } \\
\text { Postoperative: FU }\end{array}$ & $50.4 \mathrm{~Gy}$ & $50 \mathrm{~m}$ \\
\hline $\begin{array}{l}\text { Schmoll et al. }[30] \text {, } \\
\text { 2014, Germany }\end{array}$ & PETACC-6 & $\begin{array}{l}\text { Multicenter, Open-Label, Randomized, Phase } \\
\text { III }\end{array}$ & $\begin{array}{l}\text { Preoperative: } \mathrm{OX}+\mathrm{CAPE} \\
\text { Postoperative: } \mathrm{OX}+\mathrm{CAPE}\end{array}$ & $\begin{array}{l}\text { Preoperative: CAPE } \\
\text { Postoperative: CAPE }\end{array}$ & $45 \mathrm{~Gy}$ & $31 \mathrm{~m}$ \\
\hline
\end{tabular}

Abbreviations: OX, oxaliplatin; FU, fluorouracil; CAPE, capecitabine; m, month; STAR, Studio Terapia Adiuvante Retto; NSABP, National Surgical Adjuvant Breast and Bowel Project; FOWARC, FOLFOX6 Chemotherapy With or Without Radiation in Rectal Cancer; ACCORD, Actions Concertées dans les Cancers Colorectaux et Digestifs; CAO/ARO, Working Group of Surgical Oncology/Working Group of Radiation Oncology.

\section{Table 2: Baseline characteristics of the included studies of the meta-analysis}

\begin{tabular}{|c|c|c|c|c|c|c|c|c|}
\hline Studies & Arm & No. of patients & Age (years) & $\operatorname{Sex}(M / F)$ & $\begin{array}{c}\text { Clinical T Category } \\
\text { (T2/T3/T4) }\end{array}$ & $\begin{array}{c}\text { Clinical N Category } \\
\text { (N0/N1-2) }\end{array}$ & $\begin{array}{c}\text { Clinical Stage } \\
\text { (II/III) }\end{array}$ & $\begin{array}{l}\text { Location From Anal Verge }(0-5 / 5- \\
\qquad 10 />10 \mathrm{~cm})\end{array}$ \\
\hline Allegra et al. [31, 32], 2015 & $\begin{array}{c}\text { Treatment } \\
\text { Control }\end{array}$ & $\begin{array}{l}659 \\
949\end{array}$ & $\begin{array}{l}255(\geq 60) \\
414(\geq 60)\end{array}$ & $\begin{array}{l}454 / 205 \\
641 / 308\end{array}$ & $\begin{array}{l}\text { NA } \\
\text { NA }\end{array}$ & $\begin{array}{l}\text { NA } \\
\text { NA }\end{array}$ & $\begin{array}{l}406 / 253 \\
547 / 402\end{array}$ & $\begin{array}{l}130 / 149 \\
119 / 141\end{array}$ \\
\hline Aschele et al. [27], 2011 & $\begin{array}{l}\text { Treatment } \\
\text { Control }\end{array}$ & $\begin{array}{l}368 \\
379\end{array}$ & $\begin{array}{l}69(33-75) \\
63(20-75)\end{array}$ & $\begin{array}{l}245 / 123 \\
259 / 120\end{array}$ & $\begin{array}{l}17 / 300 / 50,1 \dagger \\
7 / 307 / 65\end{array}$ & $\begin{array}{c}122 / 246 \\
134 / 242,3\end{array}$ & $\begin{array}{l}\text { NA } \\
\text { NA }\end{array}$ & $\begin{array}{l}70 / 213 / 76,9 \dagger \\
89 / 202 / 81,7 \dagger\end{array}$ \\
\hline $\begin{array}{l}\text { Deng et al. [34], } \\
2016\end{array}$ & $\begin{array}{c}\text { Treatment } \\
\text { Control }\end{array}$ & $\begin{array}{l}165 \\
165\end{array}$ & $\begin{array}{l}52.2 \pm 11.8 \\
54.0 \pm 11.9\end{array}$ & $\begin{array}{l}114 / 51 \\
103 / 62\end{array}$ & $\begin{array}{l}3 / 106 / 56 \\
8 / 100 / 57\end{array}$ & $\begin{array}{l}30 / 135 \\
37 / 128\end{array}$ & $\begin{array}{l}30 / 135 \\
37 / 128\end{array}$ & $\begin{array}{l}83 / 75 / 7 \\
90 / 70 / 5\end{array}$ \\
\hline $\begin{array}{l}\text { Hong et al. }[35], \\
\text { 2014, Korea }\end{array}$ & $\begin{array}{c}\text { Treatment } \\
\text { Control }\end{array}$ & $\begin{array}{l}160 \\
161\end{array}$ & $\begin{array}{l}55(49-63) \\
54(47-61)\end{array}$ & $\begin{array}{l}118 / 42 \\
116 / 45\end{array}$ & $\begin{array}{l}24 / 133 / 3 \\
24 / 131 / 6\end{array}$ & $\begin{array}{c}58 / 102 \\
65 / 96\end{array}$ & $\begin{array}{l}\mathrm{NA} \\
\mathrm{NA}\end{array}$ & $\begin{array}{l}48 / 81 / 31 \\
45 / 89 / 27\end{array}$ \\
\hline G'erard et al. [28, 29], 2012 & $\begin{array}{c}\text { Treatment } \\
\text { Control }\end{array}$ & $\begin{array}{l}291 \\
293\end{array}$ & $\begin{array}{l}61(25-80) \\
63(34-80)\end{array}$ & $\begin{array}{c}196 / 95 \\
191 / 102\end{array}$ & $\begin{array}{l}21 / 254 / 16 \\
23 / 255 / 15\end{array}$ & $\begin{array}{l}78 / 211,2 \dagger \\
85 / 205,3 \dagger\end{array}$ & $\begin{array}{l}\mathrm{NA} \\
\mathrm{NA}\end{array}$ & $\begin{array}{l}184(0-6 \mathrm{~cm}), 107(>6 \mathrm{~cm}) \\
204(0-6 \mathrm{~cm}), 89(>6 \mathrm{~cm})\end{array}$ \\
\hline $\begin{array}{l}\text { Jiao et al. [36], } \\
2015 \text {, China }\end{array}$ & $\begin{array}{c}\text { Treatment } \\
\text { Control }\end{array}$ & $\begin{array}{l}103 \\
103\end{array}$ & $\begin{array}{l}55.8 \pm 2.5 \\
60.0 \pm 2.3\end{array}$ & $\begin{array}{l}59 / 44 \\
68 / 35\end{array}$ & $\begin{array}{l}2 / 66 / 35 \\
3 / 61 / 39\end{array}$ & $\begin{array}{l}22 / 81 \\
23 / 80\end{array}$ & $\begin{array}{l}\text { NA } \\
\text { NA }\end{array}$ & $\begin{array}{l}24 / 58 / 21 \\
25 / 57 / 21\end{array}$ \\
\hline Rödel et al. [33], 2015 & $\begin{array}{c}\text { Treatment } \\
\text { Control }\end{array}$ & $\begin{array}{l}613 \\
623\end{array}$ & $\begin{array}{l}62 \pm 10 \\
62 \pm 10\end{array}$ & $\begin{array}{l}434 / 179 \\
440 / 183\end{array}$ & $\begin{array}{l}22 / 549 / 41,1 \dagger \\
32 / 537 / 50,4 \dagger\end{array}$ & $\begin{array}{l}146 / 452,15 \dagger \\
159 / 451,13 \dagger\end{array}$ & $\begin{array}{l}146 / 452,15 \dagger \\
159 / 451,13 \dagger\end{array}$ & $\begin{array}{l}249 / 302 / 55,7 \dagger \\
216 / 336 / 64,7 \dagger\end{array}$ \\
\hline Schmoll et al. [30], 2014* & $\begin{array}{c}\text { Treatment } \\
\text { Control }\end{array}$ & $\begin{array}{l}528 \\
543\end{array}$ & $\begin{array}{l}\text { NA } \\
\text { NA }\end{array}$ & $\begin{array}{l}\text { NA } \\
\text { NA }\end{array}$ & $\begin{array}{l}\text { NA } \\
\text { NA }\end{array}$ & $\begin{array}{l}\mathrm{NA} \\
\mathrm{NA}\end{array}$ & $\begin{array}{l}\text { NA } \\
\text { NA }\end{array}$ & $\begin{array}{l}\text { NA } \\
\text { NA }\end{array}$ \\
\hline
\end{tabular}

Abbreviations: M, male; F, female; NA, not available; $\dagger$, Undetermined/data missing; * A preliminary report and later analysis of the trial were presented at the 2013 and 2014 ASCO meeting, but limited by without full-text published article.

\section{Overall grade $3-4$ toxicities}

There were 8 studies [27-36] compared the overall grade 3-4 toxicities between oxaliplatin-based regimen group and fluorouracil-based regimen group. Heterogeneity was high among the studies $(P<0.00001$, $I^{2}=83 \%$ ), so the random effect model was used to pool the outcomes. The result $(\mathrm{OR}=2.07 ; 95 \% \mathrm{CI}=1.52$ to $2.83 ; P<0.00001)$ suggested that toxicities of oxaliplatinbased regimen group were higher than that of fluorouracilbased regimen group (Figure 9).

\section{Sphincter-sparing surgery}

There were 7 studies $[27-34,36]$ compared sphincter-sparing surgery between oxaliplatin-based regimen group and fluorouracil-based regimen group. Heterogeneity was low among the studies $(P=0.39$, $I^{2}=4 \%$ ), so the fixed effect model was used to pool the outcomes. The result $(\mathrm{OR}=0.94 ; 95 \% \mathrm{CI}=0.83$ to 1.06 ; $P=0.33$ ) inferred that sphincter-sparing surgery was no significant difference between two groups (Figure 10).

\section{Publication bias}

The funnel plots were utilized to evaluate the publication bias of this meta-analysis. Funnel plot of 3-year disease-free survival (Figure 11A), funnel plot of pathologic complete response (Figure 11B) and funnel plot of overall grade 3-4 toxicities (Figure 11C) were basically inverted and funnel-shaped with bilateral symmetry, indicating that there was no obvious evidence of publication bias.

\section{DISCUSSION}

This meta-analysis compares oxaliplatin-based with fluorouracil-based neoadjuvant chemoradiotherapy and 


\begin{tabular}{|c|c|c|c|c|c|c|c|c|}
\hline Study or Subgroup & \multicolumn{2}{|c|}{ Experimental } & \multicolumn{2}{|c|}{ Control } & Weight & $\begin{array}{c}\text { Odds Ratio } \\
\text { M-H, Fixed, } 95 \% \mathrm{Cl}\end{array}$ & \multicolumn{2}{|c|}{$\begin{array}{c}\text { Odds Ratio } \\
\text { M-H, Fixed, } 95 \% \mathrm{Cl}\end{array}$} \\
\hline Allegra et al. 2015 & 467 & 643 & 474 & 641 & $25.0 \%$ & $0.93[0.73,1.20]$ & & \\
\hline Aschele et al. 2011 & 273 & 368 & 267 & 379 & $13.1 \%$ & $1.21[0.87,1.66]$ & & \\
\hline Hong et al. 2014 & 50 & 160 & 41 & 161 & $5.4 \%$ & $1.33[0.82,2.17]$ & & \\
\hline Jiao et al. 2015 & 83 & 103 & 72 & 103 & $2.7 \%$ & $1.79[0.94,3.40]$ & & \\
\hline Rödel et al. 2015 & 454 & 613 & 425 & 623 & $21.0 \%$ & $1.33[1.04,1.70]$ & & \\
\hline Schmoll et al. 2014 & 390 & 528 & 405 & 543 & $20.1 \%$ & $0.96[0.73,1.27]$ & & \\
\hline Total $(95 \% \mathrm{Cl})$ & & 2714 & & 2749 & $100.0 \%$ & $1.13[1.01,1.27]$ & & \\
\hline Total events & 1837 & & 1794 & & & & & \\
\hline $\begin{array}{l}\text { Heterogeneity: } \mathrm{Chi}^{2} \\
\text { Test for overall effec }\end{array}$ & $\begin{array}{l}7.78, \mathrm{df} \\
Z=2.0\end{array}$ & $\begin{array}{l}6(P= \\
P=0 .\end{array}$ & $\begin{array}{l}0.25) ; 1^{2} \\
04)\end{array}$ & $=23 \%$ & & & $\begin{array}{cc}0.5 & 0.7 \\
\text { Favours [experimental] }\end{array}$ & $1 \quad 1.5 \quad 2$ \\
\hline
\end{tabular}

Figure 4: 3-year disease-free survival rates of oxaliplatin-based regimen versus fluorouracil-based regimen for stage II or III rectal cancer.

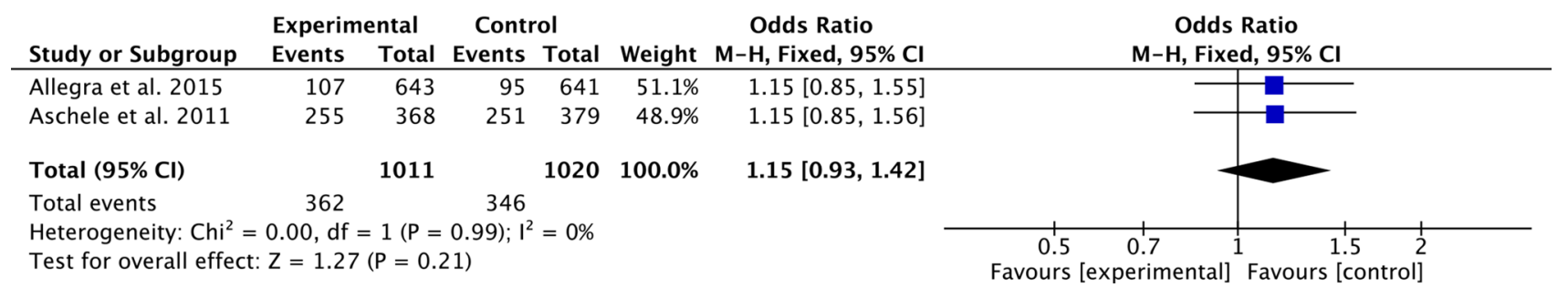

Figure 5: 5-year disease-free survival rates of oxaliplatin-based regimen versus fluorouracil-based regimen for stage II or III rectal cancer.

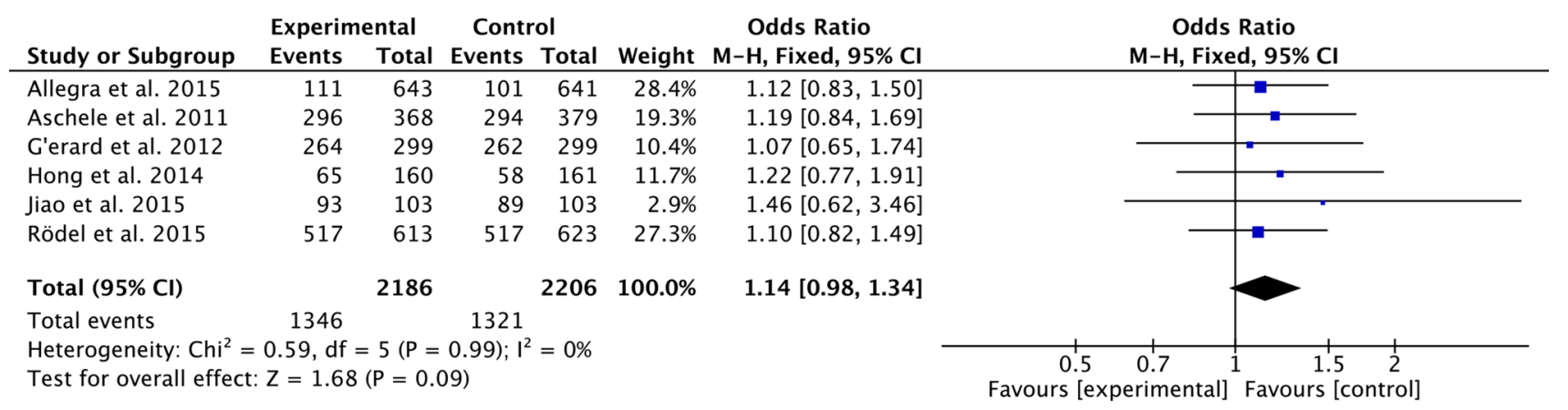

Figure 6: 3-year overall survival rates of oxaliplatin-based regimen versus fluorouracil-based regimen for stage II or III rectal cancer.

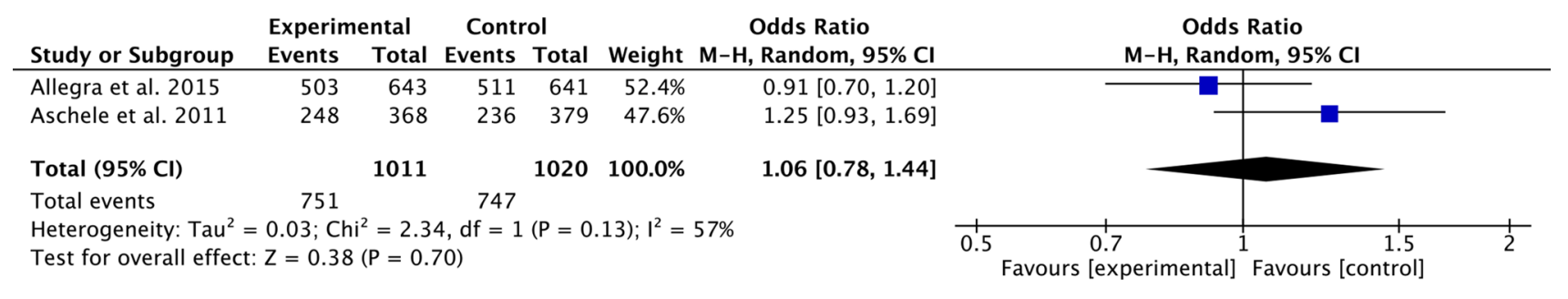

Figure 7: 5-year overall survival rates of oxaliplatin-based regimen versus fluorouracil-based regimen for stage II or III rectal cancer. 


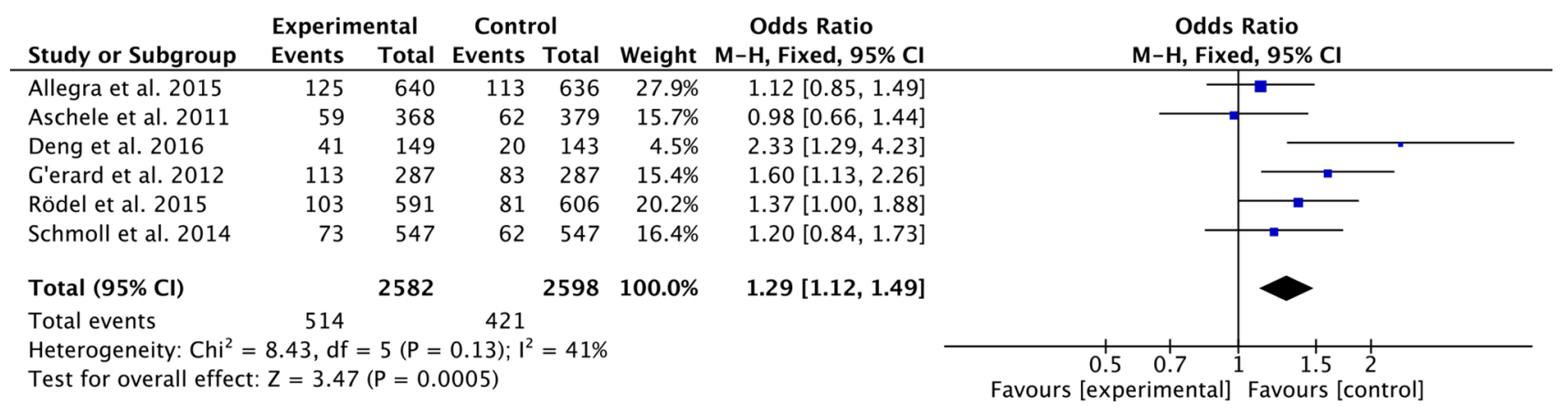

Figure 8: Pathologic complete response of oxaliplatin-based regimen versus fluorouracil-based regimen for stage II or III rectal cancer.

\begin{tabular}{|c|c|c|c|c|c|c|c|c|c|}
\hline Study or Subgroup & \multicolumn{2}{|c|}{ Experimental } & \multicolumn{2}{|c|}{ Control } & Weight & $\begin{array}{c}\text { Odds Ratio } \\
\text { M-H, Random, } 95 \% \mathrm{CI}\end{array}$ & \multicolumn{3}{|c|}{$\begin{array}{c}\text { Odds Ratio } \\
\text { M-H, Random, } 95 \% \mathrm{Cl}\end{array}$} \\
\hline Allegra et al. 2015 & 264 & 644 & 282 & 923 & $15.1 \%$ & $1.58[1.28,1.95]$ & & & $\longrightarrow$ \\
\hline Aschele et al. 2011 & 85 & 352 & 29 & 379 & $12.1 \%$ & $3.84[2.45,6.03]$ & & & \\
\hline Deng et al. 2016 & 105 & 155 & 87 & 158 & $12.0 \%$ & $1.71[1.08,2.71]$ & & & \\
\hline G'erard et al. 2012 & 74 & 291 & 32 & 293 & $12.1 \%$ & $2.78[1.77,4.37]$ & & & \\
\hline Hong et al. 2014 & 72 & 146 & 59 & 149 & $12.0 \%$ & $1.48[0.94,2.35]$ & & & \\
\hline Jiao et al. 2015 & 22 & 103 & 11 & 103 & $8.0 \%$ & $2.27[1.04,4.97]$ & & & \\
\hline Rödel et al. 2015 & 144 & 607 & 128 & 625 & $14.5 \%$ & $1.21[0.92,1.58]$ & & & \\
\hline Schmoll et al. 2014 & 201 & 547 & 83 & 547 & $14.2 \%$ & $3.25[2.43,4.35]$ & & & \\
\hline Total $(95 \% \mathrm{Cl})$ & & 2845 & & 3177 & $100.0 \%$ & $2.07[1.52,2.83]$ & & & \\
\hline Total events & 967 & & 711 & & & & & & \\
\hline $\begin{array}{l}\text { Heterogeneity: Tau }{ }^{2} \\
\text { Test for overall effec }\end{array}$ & $\begin{array}{l}0.16 ; \mathrm{Cr} \\
\mathrm{Z}=4.5\end{array}$ & $\begin{array}{l}I^{2}=40.4 \\
(P<0.0\end{array}$ & $\begin{array}{l}48, d f= \\
00001)\end{array}$ & $7(\mathrm{P}<$ & 0.00001 ); & $; I^{2}=83 \%$ & $\begin{array}{l}0.2 \\
\mathrm{Fa}\end{array}$ & $\begin{array}{l}0.5 \\
\text { xperimental] }\end{array}$ & $\begin{array}{r}2 \\
\text { Favours [cc }\end{array}$ \\
\hline
\end{tabular}

Figure 9: Overall grade 3-4 toxicities of oxaliplatin-based regimen versus fluorouracil-based regimen for stage II or III rectal cancer.

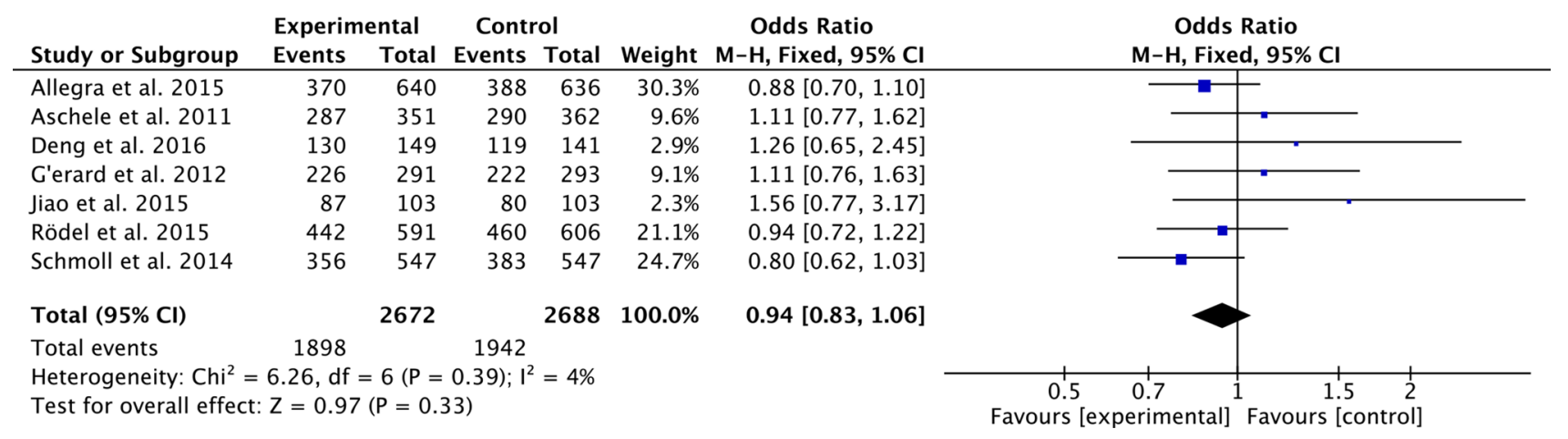

Figure 10: Sphincter-sparing surgery rates of oxaliplatin-based regimen versus fluorouracil-based regimen for stage II or III rectal cancer.

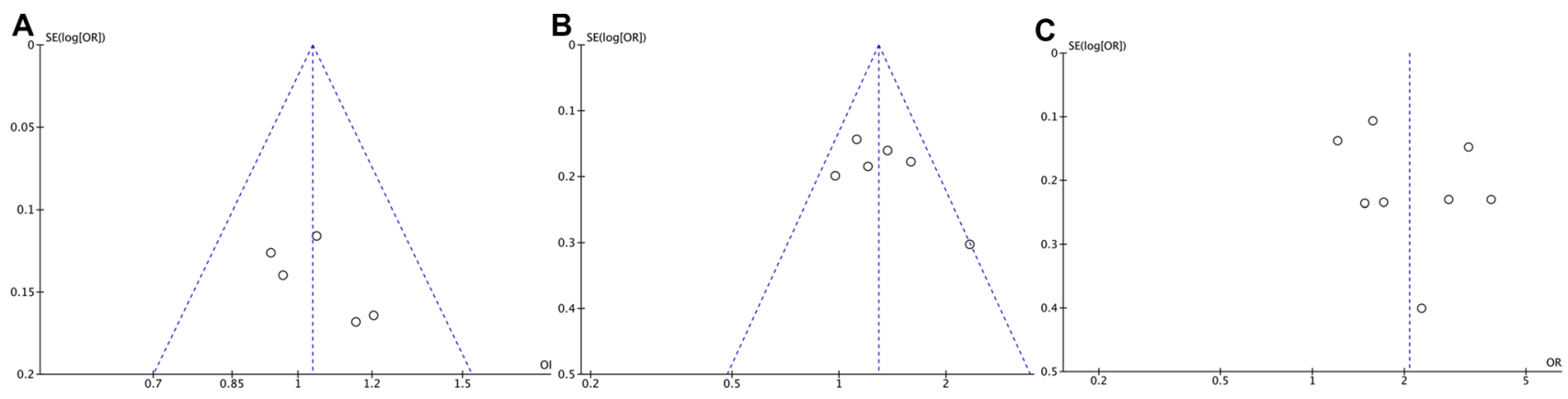

Figure 11: Funnel plots analysis of publication bias. (A) 3-year disease-free survival (B) pathologic complete response (C) overall grade $3-4$ toxicities. 
adjuvant chemotherapy for locally advanced rectal cancer. Our pooled results provide convincing evidence for attaining higher pathologic complete response and 3-year disease-free survival, but suffering greater toxicity in oxaliplatin-based regimen. Also, patients with oxaliplatinbased regimen have no improvement in sphincter-sparing surgery, 5-year disease-free survival and overall survival. Upon further analysis, it is no consensus in the real benefit of adding oxaliplatin to neoadjuvant chemoradiotherapy with fluoropyrimidine for locally advanced rectal cancer. On the other hand, patients with locally advanced rectal cancer are likely to benefit from the addition of oxaliplatin to fluoropyrimidine both neoadjuvant and adjuvant treatment. However, the pooled results should be interpreted with caution for the limitations of our study.

Several studies have recently demonstrated that neoadjuvant chemoradiotherapy enhanced the rate of sphincter-sparing surgery and local control for patients with stage II and III rectal cancers [12, 20, 37, 38]. Based on these results, neoadjuvant chemoradiotherapy has become a reference approach for patients with stage II or III rectal cancer. In our study, fluorouracil-based neoadjuvant regimen include two approach: infusion fluorouracil or oral capecitabine. The initial endpoints from NSABP R-04 trial showed that fluorouracil and capecitabine used in rectal cancer resulted in similar rates of pathologic complete response and of sphinctersparing surgery and surgical downstaging [39]. In addition, the results of NSABP R-04 trial are similar to the recently completed neoadjuvant rectal investigations $[12,20,37,38]$. These data support composite that our study analyzes infusional fluorouracil and capecitabine together in the neoadjuvant rectal setting. Nevertheless, the 5-FU control regimen was a bolus schedule in two of the larger trials [27, 33], in which the control group used a fluoropyrimidine alone. In the $\mathrm{CAO} / \mathrm{ARO} / \mathrm{AIO}-04$ study [33], oxaliplatin was given with an entirely different 5-FU schedule and a much less toxic regimen. This weakened the conclusions that adding oxaliplatin increased the overall toxicity. Because the using of a toxic control group (bolus 5-FU schedule) would diminish the ability to see the increase toxicity.

Oxaliplatin has been found to sensitize human cancer cells to the effects of radiation in vitro; in addition, several large randomized investigations demonstrate that disease-free survival was significantly enhanced by adding oxaliplatin to 5-fluorouracil in the adjuvant treatment of stage II or III colon cancer [23-26]. While the benefit of adding oxaliplatin to fluorouracil-based chemoradiation in neoadjuvant rectal cancer setting remains unclear.

Pathologic complete response is an indication of efficacy with regard to oncological outcomes. The pooled result confirms that oxaliplatin-based regimen exhibited a signicantly increased pathologic complete response rate $(\mathrm{OR}=1.29,95 \% \mathrm{CI}=1.12$ to $1.49, P=0.0005)$ than fluorouracil alone. CAO/ARO/AIO-04 [33] and FOWARC
[34] trials show that compared with fluorouracil-based agent alone, adding oxaliplatin regimen results higher pathologic complete response, and similar toxicity for patients with stage II or III rectal cancer. In contrast, the other four studies [27-32] reported increased acute toxicity without substantial improvements in pathologic complete response rates. The reasons for this are not completely understood, but might include poorer compliance as a consequence of increased toxic effects, resulting in more dose reduction and treatment interruptions [33].

To our knowledge, disease-free survival in adjuvant colorectal cancer trials and progression-free survival in metastatic cancer are commonly primary endpoints [40]. The results of our meta-analysis confirm that patients with oxaliplatin-based regimen attain higher 3-year disease-free survival, but have no improvement in 5-year disease-free survival and overall survival. Five RCTs included in this meta-analysis have disease-free survival as their primary endpoint. There are four trials, including ACCORD 12/0405-PRODIGE 2 [28, 29], STAR-0 [27], NSABP R-04 [31, 32] and PETACC-6 [30], reported no benefit of 3 or 5 years disease-free survival of oxaliplatin-based; only CAO/ARO/AIO-04 [33] trial reported 3-year diseasefree survival improved $(75.9 \%$ investigational group vs $71.2 \%$ control group, $p=0.03$ ). However, it must be noted that CAO/ARO/AIO-04 [33] and PETACC-6 [30] added oxaliplatin to both neoadjuvant chemoradiotherapy and adjuvant chemotherapy with fluorouracil-based regimen. Two further RCTs [35, 41] investigated adjuvant fluorouracil-based chemotherapy with oxaliplatin after standard preoperative fluorouracil-based preoperative chemoradiotherapy for stage II or III rectal cancer. CHRONICLE [41] trial closed prematurely because of poor patient accrual. ADORE [35] trial showed improved disease-free survival when oxaliplatin was added to adjuvant chemotherapy after preoperative fluorouracilbased chemoradiotherapy and surgery for stage II or III rectal cancer. Why an increase in the percentage of pathologic complete response do not correspond to an increase in long-term survival indicators (e.g. 5-year disease-free survival and overall survival)? One reason is the selected studies lacked long-term follow-up data for some patients and only 2 studies [27, 31] have an average follow-up time of more than 5 years. Another likely reason is pathologic complete response, as a surrogate index for curative effect, does not completely represent the longterm survival benefit for locally advanced rectal cancer.

Heterogeneity is a classical limitation of metaanalysis, and high heterogeneity may prevent educing convincing conclusions. Moderate to high heterogeneity in this meta-analysis were found only for analyses on pathologic complete response and overall grade $3+$ toxicities. Instead, no significant heterogeneity was detected for analyses on disease-free survival, overall survival and sphincter-sparing surgery. All of these increase the accuracy and reliability of the result. 
This meta-analysis has several limitations. First, stage II rectal cancer of the 8 selected studies in this meta-analysis is not further divided into low risk and high risk. Today, there is no consensus in some stage II rectal cancer on the real benefit of neoadjuvant chemotherapy (with 5-FU) followed by surgery. Stage II low risk rectal cancers probably do not need a tall chemotherapy with 5-FU or 5-FU plus oxaliplatin. Therefore, the results can be completely different according to this classification schemes. Second, data of long-term outcomes of the selected studies are deficient. Tumor locoregional relapse frequently appears at 4 or 5 years after surgery, when we used neoadjuvant chemoradiotherapy or adjuvant chemotherapy. So, the endpoints should be 5-years disease free survival and complete pathological response with a long-term follow-up. In all the 8 selected studies, only 2 studies [27, 31] show the 5-year disease free survival results and one [33] presents 3-year free survival results. Third, the radiation dose in neoadjuvant therapy as adjuvant therapy schemes, are different in the selected studies of this meta-analysis. Finally, preoperative fluorouracil-based chemotherapy regimen is diverse and the Rodel et al. [33] is the only one that proceed with oxaliplatin plus 5-Fluorouracil in the adjuvant therapy in one of branch of the trial. The ample variety of the chemotherapy schemes may jeopardize the conclusions. Therefore, the results should be interpreted with cautious due to the aforementioned limitations and further largescale, well-designed RCTs on this topic are still needed.

In conclusion, this meta-analysis compares oxaliplatin plus fluorouracil regimen with fluorouracil alone neoadjuvant chemoradiotherapy and adjuvant chemotherapy for locally advanced rectal cancer. Patients with oxaliplatinbased regimen attain higher pathologic complete response and 3-year disease-free survival, but suffer greater toxicity. Also, sphincter-sparing surgery rates, 5-year disease-free survival and overall survival are no difference between two regimens. But, the results are limited by the aforementioned limitations. Hence, the benefits of adding oxaliplatin to fluorouracil-based neoadjuvant chemoradiotherapy and adjuvant chemotherapy for locally advanced rectal cancer remains controversial, and cannot be considered a standard approach.

\section{MATERIALS AND METHODS}

This meta-analysis was conducted adhering to the PRISMA statement and the Cochrane Handbook for Systematic Reviews of Interventions (Version 5.3) to ensure data quality.

\section{Data sources and search strategy}

MEDLINE (Ovid), EMBASE and CENTRAL were searched comprehensively to identify all relevant clinical trials until January 31 2017. The bibliographies of identified articles were manually searched to identify additional studies. Ongoing clinical trials were also searched by two registers for clinical trials (www. clinicaltrials.gov, www.clinicaltrialsregister.eu).

Search strategy was MeSH terms and free-text terms, as well as variation of root words. Terms were used in different Boolean combinations within each database. The search terms included ("Rectal Neoplasms" or "rectal cancer") and ("Organoplatinum Compounds" or "oxaliplatin") and ("Radiotherapy") and (randomized controlled trial). All potentially eligible articles were retained, and then were examined to determine whether meet the inclusion criteria.

\section{Study inclusion and exclusion criteria}

Studies inclusion criteria in this meta-analysis were list as follow: (i) randomized controlled trials (RCTs); (ii) preoperative chemoradiotherapy with fluorouracil and oxaliplatin versus fluorouracil alone in locally advanced rectal cancer; preoperative chemoradiotherapy with capecitabine and oxaliplatin versus capecitabine alone in locally advanced rectal cancer. Locally advanced rectal cancer was defined as clinical (by transrectal ultrasonography and CT scan or MRI) stage II (T3-4N0) or stage III (T1-4N1-2). (iii) results describing the details of oncological outcomes and survival rate.

Studies exclusion criteria were list as follow: (i) articles without original data, such as abstracts, letters, editorials, expert opinions, case reports and reviews; (ii) studies without reporting clinical outcomes of effectiveness; (iii) studies with a sample size less than 100.

\section{Data extraction}

Data of the included studies were independently extracted by two investigators (X.F. and G.T.). Baseline characteristics included first author, year of publication, country, demographics, study design, number of patients in each arm, clinical trial information, primary endpoint, follow up, clinical disease stage, and location from anal verge. Furthermore, the following data were extracted for meta-analysis: disease-free survival, overall survival, pathologic complete response, overall grade 3-4 toxicities, and sphincter-sparing surgery. Disease-free survival was defined as the time between randomization and any of the following events: death, local relapse or distant metastasis, or second cancer, whichever occurred first. Pathologic complete response was defined as the absence of viable tumor cells in the surgical specimens. Data extracted from the included studies were checked by two other investigators (Z.R. and J.W.) to ensure accuracy and completeness.

\section{Risk of bias assessment}

Risk of bias of the included trials were independently assessed two reviewers (Z.F. and S.L.) according to the 
Cochrane Collaboration's tool. Good quality criteria studies were as follow: sequence generation randomized; allocation concealment; blinding every participant; complete outcome data; and non-selective outcome reporting. All disagreements were resolved by consensus.

\section{Statistical analysis}

The data were analyzed using Review Manager (Version 5.3 for mac). In this meta-analysis, all variables were dichotomous data, which were calculated by Odds ratio (OR) with $95 \%$ confidence intervals (CI). If $95 \%$ CI of OR did not include the value $1, P<10.05$ was considered to be statistically significant.

Heterogeneity was evaluated by the degree of inconsistency $\left(I^{2}\right)$ and $P$ value to assess the variation across studies. If $I^{2}>50 \%$ and $P<0.05$, a random effect model was used. Otherwise, data were pooled using a fixed effect model. $P<0.05$ was considered as statistical significance in the integration results. Publication bias was analyzed using a funnel plot for standard error by effect size (log OR).

\section{CONFLICTS OF INTEREST}

The authors have declared that no competing interests exist.

\section{GRANT SUPPORT}

This project is supported by Science and Technology Project Foundation of Nanjing (Grant no: 201303032); Medical Science and Technology Development Foundation of Jiangsu University (Grant no: JLY20140160).

\section{REFERENCES}

1. Ferlay J, Steliarova-Foucher E, Lortet-Tieulent J, Rosso S, Coebergh JW, Comber H, Forman D, Bray F. Cancer incidence and mortality patterns in Europe: estimates for 40 countries in 2012. Eur J Cancer. 2013; 49:1374-1403.

2. Brenner H, Bouvier AM, Foschi R, Hackl M, Larsen IK, Lemmens V, Mangone L, Francisci S, Group EW. Progress in colorectal cancer survival in Europe from the late 1980s to the early 21st century: the EUROCARE study. Int J Cancer. 2012; 131:1649-1658.

3. Siegel RL, Miller KD, Jemal A. Cancer Statistics, 2017. CA Cancer J Clin. 2017; 67:7-30.

4. McCourt M, Armitage J, Monson JR. Rectal cancer. Surgeon. 2009; 7:162-169.

5. Maringe C, Walters S, Rachet B, Butler J, Fields T, Finan P, Maxwell R, Nedrebo B, Pahlman L, Sjovall A, Spigelman A, Engholm G, Gavin A, et al. Stage at diagnosis and colorectal cancer survival in six high-income countries: a population-based study of patients diagnosed during 2000-2007. Acta Oncol. 2013; 52:919-932.
6. Shen L, van Soest J, Wang J, Yu J, Hu W, Gong YU, Valentini V, Xiao Y, Dekker A, Zhang Z. Validation of a rectal cancer outcome prediction model with a cohort of Chinese patients. Oncotarget. 2015; 6:38327-38335. doi: 10.18632/oncotarget.5195.

7. George TJ JR, Allegra CJ, Yothers G. Neoadjuvant Rectal (NAR) Score: a New Surrogate Endpoint in Rectal Cancer Clinical Trials. Curr Colorectal Cancer Rep. 2015; 11:275-280.

8. van Gijn W, Marijnen CA, Nagtegaal ID, Kranenbarg EM, Putter H, Wiggers T, Rutten HJ, Pahlman L, Glimelius B, van de Velde CJ, Dutch Colorectal Cancer G. Preoperative radiotherapy combined with total mesorectal excision for resectable rectal cancer: 12-year follow-up of the multicentre, randomised controlled TME trial. Lancet Oncol. 2011; 12:575-582.

9. Sebag-Montefiore D, Stephens RJ, Steele R, Monson J, Grieve R, Khanna S, Quirke P, Couture J, de Metz C, Myint AS, Bessell E, Griffiths G, Thompson LC, et al. Preoperative radiotherapy versus selective postoperative chemoradiotherapy in patients with rectal cancer (MRC CR07 and NCIC-CTG C016): a multicentre, randomised trial. Lancet. 2009; 373:811-820.

10. Folkesson J, Birgisson H, Pahlman L, Cedermark B, Glimelius B, Gunnarsson U. Swedish Rectal Cancer Trial: long lasting benefits from radiotherapy on survival and local recurrence rate. J Clin Oncol. 2005; 23:5644-5650.

11. McCarthy K, Pearson K, Fulton R, Hewitt J. Pre-operative chemoradiation for non-metastatic locally advanced rectal cancer. Cochrane Database Syst Rev. 2012; 12:CD008368.

12. Sauer R, Liersch T, Merkel S, Fietkau R, Hohenberger W, Hess C, Becker H, Raab HR, Villanueva MT, Witzigmann H, Wittekind C, Beissbarth T, Rodel C. Preoperative versus postoperative chemoradiotherapy for locally advanced rectal cancer: results of the German $\mathrm{CAO} / \mathrm{ARO} / \mathrm{AIO}-94$ randomized phase III trial after a median follow-up of 11 years. J Clin Oncol. 2012; 30:1926-1933.

13. Janjan NA, Crane C, Feig BW, Cleary K, Dubrow R, Curley S, Vauthey JN, Lynch P, Ellis LM, Wolff R, Lenzi R, Abbruzzese J, Pazdur R, et al. Improved overall survival among responders to preoperative chemoradiation for locally advanced rectal cancer. Am J Clin Oncol. 2001; 24:107-112.

14. Fietkau R, Barten M, Klautke G, Klar E, Ludwig K, Thomas H, Brinckmann W, Friedrich A, Prall F, Hartung G, Kuchenmeister U, Kundt G. Postoperative chemotherapy may not be necessary for patients with ypN0-category after neoadjuvant chemoradiotherapy of rectal cancer. Dis Colon Rectum. 2006; 49:1284-1292.

15. Bujko K, Glynne-Jones R, Bujko M. Does adjuvant fluoropyrimidine-based chemotherapy provide a benefit for patients with resected rectal cancer who have already received neoadjuvant radiochemotherapy? A systematic review of randomised trials. Ann Oncol. 2010; 21:1743-1750.

16. Kiran RP, Kirat HT, Burgess AN, Nisar PJ, Kalady MF, Lavery IC. Is adjuvant chemotherapy really needed after 
curative surgery for rectal cancer patients who are nodenegative after neoadjuvant chemoradiotherapy? Ann Surg Oncol. 2012; 19:1206-1212.

17. Khrizman P, Niland JC, ter Veer A, Milne D, Bullard Dunn K, Carson WE 3rd, Engstrom PF, Shibata S, Skibber JM, Weiser MR, Schrag D, Benson AB 3rd. Postoperative adjuvant chemotherapy use in patients with stage II/III rectal cancer treated with neoadjuvant therapy: a national comprehensive cancer network analysis. J Clin Oncol. 2013; 31:30-38.

18. Breugom AJ, Swets M, Bosset JF, Collette L, Sainato A, Cionini L, Glynne-Jones R, Counsell N, Bastiaannet E, van den Broek CB, Liefers GJ, Putter H, van de Velde CJ. Adjuvant chemotherapy after preoperative (chemo) radiotherapy and surgery for patients with rectal cancer: a systematic review and meta-analysis of individual patient data. Lancet Oncol. 2015; 16:200-207.

19. Bujko K, Glimelius B, Valentini V, Michalski W, Spalek M. Postoperative chemotherapy in patients with rectal cancer receiving preoperative radio(chemo)therapy: A metaanalysis of randomized trials comparing surgery $+/-$ a fluoropyrimidine and surgery + a fluoropyrimidine $+/-$ oxaliplatin. Eur J Surg Oncol. 2015; 41:713-723.

20. Gerard JP, Conroy T, Bonnetain F, Bouche O, Chapet O, Closon-Dejardin MT, Untereiner M, Leduc B, Francois E, Maurel J, Seitz JF, Buecher B, Mackiewicz R, et al. Preoperative radiotherapy with or without concurrent fluorouracil and leucovorin in T3-4 rectal cancers: results of FFCD 9203. J Clin Oncol. 2006; 24:4620-4625.

21. O'Connell MJ, Martenson JA, Wieand HS, Krook JE, Macdonald JS, Haller DG, Mayer RJ, Gunderson LL, Rich TA. Improving adjuvant therapy for rectal cancer by combining protracted-infusion fluorouracil with radiation therapy after curative surgery. N Engl J Med. 1994; 331:502-507.

22. van de Velde CJ, Boelens PG, Borras JM, Coebergh JW, Cervantes A, Blomqvist L, Beets-Tan RG, van den Broek CB, Brown G, Van Cutsem E, Espin E, Haustermans K, Glimelius B, et al. EURECCA colorectal: multidisciplinary management: European consensus conference colon \& rectum. Eur J Cancer. 2014; 50:1 e1-1 e34.

23. Andre $\mathrm{T}$, Boni C, Mounedji-Boudiaf L, Navarro M, Tabernero J, Hickish T, Topham C, Zaninelli M, Clingan P, Bridgewater J, Tabah-Fisch I, de Gramont A, Multicenter International Study of Oxaliplatin/5-Fluorouracil/Leucovorin in the Adjuvant Treatment of Colon Cancer I. Oxaliplatin, fluorouracil, and leucovorin as adjuvant treatment for colon cancer. N Engl J Med. 2004; 350:2343-2351.

24. Yothers G, O'Connell MJ, Allegra CJ, Kuebler JP, Colangelo LH, Petrelli NJ, Wolmark N. Oxaliplatin as adjuvant therapy for colon cancer: updated results of NSABP C-07 trial, including survival and subset analyses. J Clin Oncol. 2011; 29:3768-3774.

25. Andre $\mathrm{T}$, de Gramont A, Vernerey D, Chibaudel B, Bonnetain F, Tijeras-Raballand A, Scriva A, Hickish T, Tabernero J, Van Laethem JL, Banzi M, Maartense E,
Shmueli E, et al. Adjuvant Fluorouracil, Leucovorin, and Oxaliplatin in Stage II to III Colon Cancer: Updated 10Year Survival and Outcomes According to BRAF Mutation and Mismatch Repair Status of the MOSAIC Study. J Clin Oncol. 2015; 33:4176-4187.

26. Andre $\mathrm{T}$, Boni $\mathrm{C}$, Navarro $\mathrm{M}$, Tabernero J, Hickish T, Topham C, Bonetti A, Clingan P, Bridgewater J, Rivera F, de Gramont A. Improved overall survival with oxaliplatin, fluorouracil, and leucovorin as adjuvant treatment in stage II or III colon cancer in the MOSAIC trial. J Clin Oncol. 2009; 27:3109-3116.

27. Aschele C, Cionini L, Lonardi S, Pinto C, Cordio S, Rosati G, Artale S, Tagliagambe A, Ambrosini G, Rosetti P, Bonetti A, Negru ME, Tronconi MC, et al. Primary tumor response to preoperative chemoradiation with or without oxaliplatin in locally advanced rectal cancer: pathologic results of the STAR-01 randomized phase III trial. Journal of clinical oncology. 2011; 29:2773-2780.

28. Gérard JP, Azria D, Gourgou-Bourgade S, Martel-Lafay I, Hennequin C, Etienne PL, Vendrely V, François E, de La Roche G, Bouché O, Mirabel X, Denis B, Mineur L, et al. Clinical outcome of the ACCORD 12/0405 PRODIGE 2 randomized trial in rectal cancer. Journal of clinical oncology. 2012; 30:4558-4565.

29. Gérard JP, Azria D, Gourgou-Bourgade S, Martel-Laffay I, Hennequin C, Etienne PL, Vendrely V, François E, de La Roche G, Bouché O, Mirabel X, Denis B, Mineur L, et al. Comparison of two neoadjuvant chemoradiotherapy regimens for locally advanced rectal cancer: results of the phase III trial ACCORD 12/0405-Prodige 2. Journal of clinical oncology. 2010; 28:1638-1644.

30. Schmoll HJ, Haustermans K, Price TJ, Nordlinger B, Hofheinz R, Daisne JF, Janssens J, Brenner B, Schmidt P, Reinel H, Hollerbach S, Caca K, Fauth FWB, et al. Preoperative chemoradiotherapy and postoperative chemotherapy with capecitabine and oxaliplatin versus capecitabine alone in locally advanced rectal cancer: Disease-free survival results at interim analysis. Journal of clinical oncology. 2014.

31. Allegra CJ, Yothers G, O’Connell MJ, Beart RW, Wozniak TF, Pitot HC, Shields AF, Landry JC, Ryan DP, Arora A, Evans LS, Bahary N, Soori G, et al. Neoadjuvant 5-FU or Capecitabine Plus Radiation With or Without Oxaliplatin in Rectal Cancer Patients: A Phase III Randomized Clinical Trial. Journal of the National Cancer Institute. 2015; 107:djv248.

32. O'Connell MJ, Colangelo LH, Beart RW, Petrelli NJ, Allegra CJ, Sharif S, Pitot HC, Shields AF, Landry JC, Ryan DP, Parda DS, Mohiuddin M, Arora A, et al. Capecitabine and oxaliplatin in the preoperative multimodality treatment of rectal cancer: surgical end points from National Surgical Adjuvant Breast and Bowel Project trial R-04. Journal of clinical oncology. 2014; 32:1927-1934.

33. Rödel C, Graeven U, Fietkau R, Hohenberger W, Hothorn T, Arnold D, Hofheinz RD, Ghadimi M, Wolff HA, Lang- 
Welzenbach M, Raab HR, Wittekind C, Ströbel P, et al. Oxaliplatin added to fluorouracil-based preoperative chemoradiotherapy and postoperative chemotherapy of locally advanced rectal cancer (the German CAO/ARO/AIO-04 study): final results of the multicentre, open-label, randomised, phase 3 trial. The Lancet Oncology. 2015; 16:979-989.

34. Deng Y, Chi P, Lan P, Wang L, Chen W, Cui L, Chen D, Cao J, Wei H, Peng X, Huang Z, Cai G, Zhao R, et al. Modified FOLFOX6 With or Without Radiation Versus Fluorouracil and Leucovorin With Radiation in Neoadjuvant Treatment of Locally Advanced Rectal Cancer: Initial Results of the Chinese FOWARC Multicenter, Open-Label, Randomized Three-Arm Phase III Trial. Journal of clinical oncology. 2016; 34:3300-3307.

35. Hong YS, Nam BH, Kim KP, Kim JE, Park SJ, Park YS, Park JO, Kim SY, Kim TY, Kim JH, Ahn JB, Lim SB, Yu CS, et al. Oxaliplatin, fluorouracil, and leucovorin versus fluorouracil and leucovorin as adjuvant chemotherapy for locally advanced rectal cancer after preoperative chemoradiotherapy (ADORE): an open-label, multicentre, phase 2, randomised controlled trial. Lancet Oncol. 2014; 15:1245-1253.

36. Jiao D, Zhang R, Gong Z, Liu F, Chen Y, Yu Q, Sun L, Duan H, Zhu S, Liu F, Wang J, Jia J. Fluorouracil-based preoperative chemoradiotherapy with or without oxaliplatin for stage II/III rectal cancer: a 3-year follow-up study. Chin J Cancer Res. 2015; 27:588-596.

37. Bosset JF, Collette L, Calais G, Mineur L, Maingon P, Radosevic-Jelic L, Daban A, Bardet E, Beny A, Ollier JC,
Trial ERG. Chemotherapy with preoperative radiotherapy in rectal cancer. N Engl J Med. 2006; 355:1114-1123.

38. Roh MS, Colangelo LH, O'Connell MJ, Yothers G, Deutsch M, Allegra CJ, Kahlenberg MS, Baez-Diaz L, Ursiny CS, Petrelli NJ, Wolmark N. Preoperative multimodality therapy improves disease-free survival in patients with carcinoma of the rectum: NSABP R-03. J Clin Oncol. 2009; 27:5124-5130.

39. O’Connell MJ, Colangelo LH, Beart RW, Petrelli NJ, Allegra CJ, Sharif S, Pitot HC, Shields AF, Landry JC, Ryan DP, Parda DS, Mohiuddin M, Arora A, et al. Capecitabine and oxaliplatin in the preoperative multimodality treatment of rectal cancer: surgical end points from National Surgical Adjuvant Breast and Bowel Project trial R-04. Journal of clinical oncology. 2014; 32:1927-1934.

40. Li J, Liu H, Hu J, Liu S, Yin J, Du F, Yuan J, Lv B. New tumor regression grade for rectal cancer after neoadjuvant therapy and radical surgery. Oncotarget. 2015; 6:42222-42231. doi: 10.18632/oncotarget.6008.

41. Glynne-Jones R, Counsell N, Quirke P, Mortensen N, Maraveyas A, Meadows HM, Ledermann J, SebagMontefiore D. Chronicle: results of a randomised phase III trial in locally advanced rectal cancer after neoadjuvant chemoradiation randomising postoperative adjuvant capecitabine plus oxaliplatin (XELOX) versus control. Ann Oncol. 2014; 25:1356-1362. 